\title{
Política y prensa catalanistas en Buenos Aires: la inmigración y el compromiso ideológico en las «Glosses Femenines» de Gràcia Bassa de Llorens*
}

\author{
Catalanist politics and media in Buenos Aires: immigration \\ and ideological commitment in the «Glosses Femenines» \\ by Gràcia Bassa de Llorens
}

\author{
Marcela Lucci \\ ORCID iD: https://orcid.org/0000-0003-1295-4701 \\ Universitat de Girona
}

La colectividad catalana de Buenos Aires se caracterizó por su heterogeneidad. Los «catalanes de América» porteños se destacaron por su compromiso cultural y político catalanista, que difundían a través del periodismo. Analizaremos el pensamiento de la docente, periodista, folclorista y poeta catalanista Gràcia Bassa de Llorens en las «Glosses Femenines» que publicó entre 1917 y 1925 en la revista Ressorgiment de Buenos Aires para indagar en la manera en que fomentaron el compromiso político femenino y difundieron el imaginario catalanista entre la colectividad catalana de América.

Palabras Clave: catalanismo separatista radical de ultramar; Gràcia Bassa de Llorens; «Glosses Femenines»; mujeres periodistas; revista Ressorgiment.

The Catalan community of Buenos Aires stood out for its heterogeneity. The "Catalans of America» of Buenos Aires stood out for their Catalan cultural and political commitment, disseminated through journalism. This article analyses the thought of the Catalan teacher, journalist, folklorist and poet Gràcia Bassa de Llorens in the «Glosses Femenines» she published between 1917 and 1925 in the magazine Ressorgiment of Buenos Aires to examine how they fostered female political commitment and disseminated the Catalanist imaginary among the Catalan community of America.

KeYwords: radical separatist Overseas Catalanism; Gràcia Bassa de Llorens; «Glosses Femenines»; women journalists; Ressorgiment Magazine.

Copyright: (C) 2020 CSIC. Este es un artículo de acceso abierto distribuido bajo los términos de la licencia de uso y distribución Creative Commons Reconocimiento 4.0 Internacional (CC BY 4.0).

* Proyecto «La patria hispana, la raza latina. Intelectuales, identidades colectivas y proyectos políticos entre España, Italia y Argentina (1880-1945)» (HAR2016-75324-P). 
Ya sabemos que la cuestión principal es, siempre, el buen nombre de Cataluña. $Y$ en mantener, por virtud de nuestros afanes, bien alto el concepto que de ella tengan nuestros hijos, y que deseen conocerla y amarla, consisten las obligaciones de los que estamos alejados de ella. ${ }^{1}$

\section{Introducción}

Según sus propias palabras, en 1907 Maria Gràcia Bassa i Rocas viajó desde Barcelona a la Argentina junto con su esposo Joan Llorens i Carreras pensando que volvería al cabo de dos años. ${ }^{2}$ Falleció en 1961 en Buenos Aires y, salvo las dos visitas que realizó en 1918 y 1934, nunca regresó definitivamente a Cataluña. ${ }^{3}$ Sin embargo, la distancia y a veces el aislamiento geográfico - durante muchos años la pareja vivió en Trenque Lauquen, una pequeña ciudad bonaerense distante $420 \mathrm{~km}$ de Buenos Aires y comunicada con la capital solamente mediante caminos de tierra y, desde 1895, por el ferrocarril $1^{4}$ — no restringieron su vinculación con la cultura catalana. Por el contrario, los textos de Gràcia Bassa se convirtieron en el referente femenino catalanista más activo del continente americano durante más de cuarenta años. Entre 1917 y 1960 publicó en la porteña Ressorgiment —la revista mensual vocera del separatismo radical ultramarino fundada en 1916 por Pius Àrias, Manel Cairol, Francesc Colomer y quien sería su director hasta 1972, Hipòlit Nadal i Mallol — casi dos centenares de artículos periodísticos, más de doscientos cuarenta poemas y alrededor de cien trabajos tales como traducciones, semblanzas biográficas y críticas artísticas y literarias. Entre ellos, las «Glosses Femenines», que firmaría como Alidé y aparecieron en cada número de Ressorgiment entre 1917 y 1925, constituyen su aportación más vinculada a la opinión pública catalanista femenina.

La cercanía de Bassa a una tarea intelectual no era casual ni aislada. Facilitada por el auge finisecular de las comunicaciones, la práctica del periodismo como forma de participación política — sumada a la socialización en espacios públicos caracterizada por una «auto-organización»en

1 Bassa de Llorens [Gràcia B. de Llorens], «El tema planyívol», Ressorgiment, 365, Buenos Aires, 1946, 5932. Todas las traducciones de artículos de Ressorgiment han sido efectuadas por la autora.

2 Bassa de Llorens [Gràcia B. de Llorens], «Els fets gloriosos», Ressorgiment, 490, Buenos Aires, 1957, 7891.

3 Bacardí, 2016, 19-30.

4 Mignone, 2013, 19.

5 Lucci, 2014. 
clubes y asociaciones - era esencial en el catalanismo peninsular y había devenido en uno de sus vehículos de difusión cultural predilectos durante la eclosión de la Renaixença a mediados del siglo XIX:

Es en este contexto que se construirá una opinión pública que ya podemos calificar de catalana [...] Este es un fenómeno importantísimo de socialización, de vinculación y de incremento de todo tipo de relaciones [...] que servirá para la creación de una opinión pública catalana, ya no solo local o comarcal. Así se catalanizan los problemas porque la gente puede estar al día de lo que se discute y decide en Barcelona, y ya no deben esperar tres o cuatro días para enterarse, como pasaba antes. ${ }^{6}$

El empuje de la prensa catalana estaba integrado en la vitalidad del periodismo español finisecular. ${ }^{7}$ Para comienzos del novecientos, además, este florecimiento había facilitado la entrada de la mujer en la vida cultural peninsular a partir del ejercicio de una profesión que diversificaba su atención respecto del ámbito familiar: «La prensa española, en concreto, ve multiplicarse las revistas femeninas desde mediados del siglo XIX; además asiste a la eclosión del periodismo femenino entre 1850 y 1936, y muestra ya a principios del siglo $\mathrm{XX}$, la existencia de colaboradoras fijas en determinadas secciones que albergan los grandes diarios». ${ }^{8}$ Los artículos de Emilia Pardo Bazán, Isabel Oyarzábal, Concepción Gimeno o Carmen de Burgos - considerada la primera mujer que se desempeñó como corresponsal de guerra' ${ }^{9}$ - introdujeron con voz propia las premisas del pensamiento feminista en el país, reflejaron la coyuntura europea desde una perspectiva femenina presente cada vez de manera más sistemática, y constituyeron una vía legítima de integración y protagonismo de la mujer en los asuntos políticos, sociales y culturales de la sociedad española.

En el contexto peninsular del periodismo de masas que acabamos de apuntar, la posibilidad de la prensa catalana de expresarse en su propio idioma constituyó un valor agregado central. Al ser percibido tradicionalmente como componente fundacional de la catalanidad, el lenguaje se potenció como elemento clave para analizar la dicotomía centro/periferia peninsular, ya que permitía exponer ante la opinión pública las «reivindicaciones de las culturas minoritarias contra la hegemonía del poder político y cultural» ${ }^{10}$ que caracterizaban la problemática de la construcción de la identidad

6 Riquer, 1996, 19.

7 Louis, 2018, 34-35.

8 Servén Díez, 2012, 1062-1063.

9 Louis, 2018, 34

10 Thiese, 2013, 16. 
española durante la Restauración. Así, la lengua catalana traspasó el ámbito privado, ganó espacios en los medios de comunicación, se reforzó como herramienta de socialización y rebatió con acento propio la cosmovisión identitaria española en la cual el castellano era percibido como el componente medular, dotado de una «carga cultural» $»^{11} \tan$ natural que soslayaba la aportación de los acervos de las restantes nacionalidades ibéricas.

Durante la segunda mitad del siglo XIX, sin embargo, la cosmovisión estimulada por los círculos intelectuales y políticos de la burguesía catalanista consideraba que la mujer no debía abandonar del todo la égida familiar ni la función de educadora de las jóvenes generaciones: la política catalana aceptaba un feminismo burgués con un modelo de mujer moderna que podía acceder a la formación y al mundo laboral, pero que no debía perder en absoluto los valores de la femineidad que marcaba el discurso vigente ni su «naturaleza» y objetivo principal, que se encontraba dentro del ámbito del hogar. ${ }^{12}$ Esta posición incluía al periodismo: «Que la mujer pusiera su firma al pie de un artículo periodístico escapaba a cualquier viso de normalidad». ${ }^{13}$ No obstante, y debido a que para la Renaixença la preservación y la difusión cultural sí formaban parte de los principales objetivos de la mujer catalana educada en cánones de vanguardia ${ }^{14}$ — que se desarrollaban de manera específica en el folclorismo-, se abrieron nuevos espacios culturales desde los cuales comenzó a vincularse con la esfera pública ${ }^{15}$ La impronta femenina en la prensa catalana comenzó a ser evidente: desde proyectos editoriales como Or i Grana o Feminal, y los escritos de Francesca Bonnemaison o Carme Karr, se ocupaba no solo de la temática considerada propia de la mujer, sino también de cuestiones sociales y políticas derivadas de la coyuntura del problema catalán. ${ }^{16}$

El particular activismo cívico catalanista peninsular que se canalizó a través del periodismo - y que se integraría, desde el último tercio del ochocientos, con la militancia política- se verificó también en las prácticas de los «catalanes de América», el sector de la colectividad catalana que se adhirió a esa teoría política al asentarse en el Nuevo Mundo desde la etapa conocida como de emigración masiva. ${ }^{17}$ Desde finales del siglo XIX, las

11 Archilés, 2018, 10.

12 González i Vilalta, 2006, 6.

13 Canyà, 1933, 50.

14 Llopart y Ros, 2012, 43.

15 Palau, 2012, 17.

16 Idem.

17 Lucci, 2010. 
asociaciones voluntarias que reivindicaban la filiación catalanista lo hacían de diferentes maneras. Estos «espacios sociales complejos» ${ }^{18}$ podían justificar a partir de ella su acción cultural y social o enunciarla en sus estatutos, como el Centre Catalanista de Santiago de Cuba, su homónimo de Santiago de Chile o el Casal Català de Buenos Aires al que pertenecían los fundadores de Ressorgiment ${ }^{19}$ podían utilizarla para respaldar la paulatina inclusión de acción política en sus actividades habituales, como es el caso del Centre Català de Mendoza (Argentina) ${ }^{20} \mathrm{y}$ también podían adherirse a ella para legitimar expresos objetivos políticos, como es el caso del Catalunya Grop Nacionalista Radical de La Habana (Cuba) y del Comité Llibertat de Buenos Aires. ${ }^{21}$ Pero todas estas entidades vieron reforzada su acción en la sistemática difusión de una identidad nacional propia legitimada por un acervo cultural en común, que se llevaba a cabo —en español o en catalán— desde las publicaciones periódicas que se editaban en América. ${ }^{22}$

Para 1917, cuando Bassa comenzó a colaborar en la revista que dirigía Nadal desde hacía unos meses, la prensa étnica catalanista porteña tenía en su haber, entre otros, los antecedentes de L'Aureneta — primera publicación americana en idioma catalán que había fundado en 1876 Antoni de Paula Aleu, que no se adhería al separatismo pero sí a los postulados catalanistas y que se editó hasta 1899-, Catalunya al Plata — periódico independiente que apareció durante nueve meses desde abril de 1909—, o Catalunya Nova, que se publicó durante 1914. ${ }^{23}$

La transición desde Gràcia Bassa — quintaescencia del modelo catalanista de mujer moderna, educada y comprometida con su cultura - hasta Alidé - la primera personificación de la presencia femenina más constante del pensamiento separatista ultramarino - no ha sido estudiada, como tampoco lo ha sido en exclusividad el resto de su producción periodística en Ressorgiment. Su figura ha promovido estudios específicos, entre los que debemos destacar Gràcia Bassa, poeta, periodista i traductora, de Montserrat Bacardí, ${ }^{24}$ que se ocupa de su derrotero intelectual en el contexto de la coyuntura catalana, o el de Montserrat Palau, «Les aportacions

18 Jofre Cabello, 2000, 49

19 Lucci, 2015a.

20 Lucci, 2016.

21 Lucci, 2010. Ruiz García, 2015.

22 Lucci, 2010.

23 Lucci, 2010. Fernández, 2016.

24 Bacardí, 2016. 
al feminisme de les folkloristes Maria Gràcia Bassa i Maria Baldó», ${ }^{25}$ que analiza su contribución al campo del folclore. Sin embargo, la etapa argentina de la periodista catalana no ha despertado un interés central, a pesar de la envergadura de su presencia en la vertiente americana del pensamiento catalanista. Efectivamente, la aportación de Gràcia Bassa al discurso del separatismo porteño permite integrar los estudios migratorios en enfoques teóricos novedosos que relacionan las problemáticas de la emigración y del exilio con aspectos que coadyuvan a dejar atrás puntos de vista más tradicionales, para centrarse en cuestiones poco trabajadas hasta el momento. Por un lado, en la propia historiografía sobre el catalanismo separatista radical de ultramar, ya que esta perspectiva —introducida en los estudios socioculturales de las migraciones peninsulares hacia América solamente desde comienzos del siglo XXI - carece todavía de trabajos que estudien el rol de las mujeres y evalúen el peso de su acción en el campo social, cultural y político a lo largo del novecientos, no solo en la Argentina, sino en el entorno americano en general. Por otra parte, estudiar a Alidé como primera aproximación a la carrera intelectual de Bassa en el separatismo ultramarino permite desarrollar investigaciones centradas en cuestiones de género para profundizar en las estrategias migratorias femeninas y estudiar las tácticas del colectivo femenino catalán - y catalanista - no ya como «elemento principal de las reagrupaciones familiares, de la puesta en acción y del mantenimiento de las redes migratorias», ${ }^{26}$ sino también como agentes de difusión ideológica y cultural en la esfera pública. En ese sentido, esta perspectiva defiende también la pertinencia de analizar la producción periodística de Gràcia Bassa a partir de la imbricación de los estudios sobre género y sobre nación, «desde los planteamientos propios de una nueva historia cultural de la política que atiende a la relevancia del análisis de los discursos y de la construcción de identidades».$^{27}$ De esta manera, desde un caso testigo y a partir de propuestas teóricas renovadoras, es posible abordar de manera más rigurosa la complejidad de la vida política española contemporánea y, en nuestro caso, su impacto en el colectivo que se asentó en América durante el siglo XX.

Desde la originalidad y la pertinencia teóricas apuntadas, efectuaremos un primer estudio específico de una parte de la producción intelectual de Gràcia Bassa en Ressorgiment de Buenos Aires. En esta oportunidad,

25 Palau, 2012, 13-25

26 Ortuño Martínez, 2012, 2.

27 Miralles, 2017, 35. 
nos centraremos exclusivamente en la columna «Glosses Femenines» que, con el seudónimo de Alidé, publicó regularmente desde 1917 hasta 1925, dejando de lado el resto de su copiosa labor periodística, su producción poética y los escritos que dedicó a las semblanzas biográficas, a las traducciones y a las críticas literarias. Este recorte nos permitirá concentrar nuestra atención en la perspectiva cultural y política de su pensamiento en el contexto de la intelectualidad que adhería al separatismo en la Argentina y en la manera en que desarrolló una visión femenina de los asuntos de su tierra natal y del papel que les tocaba jugar en el futuro de Cataluña a las catalanas que habían emigrado o se habían exiliado en el Nuevo Mundo. El conjunto de las «Glosses» nos permitirá acercarnos a un corpus inédito que recalca la importancia de las fuentes hemerográficas o literarias - que comportan la inclusión de la interdisciplinariedad y la atención a la subjetividad individual - para un sólido análisis metodológico de los procesos históricos: «aceptar que el conocimiento histórico tiene una dimensión subjetiva inevitable o que se transmite a través de estructuras y recursos narrativos diversos, no implica que se pueda desdibujar la pretensión de verdad de la disciplina histórica — de explicar lo que realmente ocurrió y por qué». ${ }^{28}$ Así, prestaremos atención al pensamiento de Alidé, indagaremos en los cambios temáticos y formales que haya experimentado a lo largo de los años y estableceremos la relevancia de su aportación a la cosmovisión identitaria nacional del colectivo catalanista ultramarino en general y de la propia Gràcia Bassa en particular. Indagaremos en su discurso para atender a la vertiente feminista de sus escritos con el fin de identificar sus orígenes teóricos, de estudiar la manera en que delineó sus objetivos intelectuales y de rastrear la influencia que ejerció en el papel que otorgaba a la mujer catalana comprometida con ideales nacionalistas.

\section{Alidé: un seudónimo para el feminismo catalanista}

La labor periodística de Gràcia Bassa en la Argentina fue un camino casi natural que siguió a la actividad cultural y docente que había desarrollado en Cataluña. Los recuerdos de su madre, la prestigiosa folclorista Irene Rocas, indican el beneplácito con que se recibían las inclinaciones intelectuales de la primogénita de la familia. ${ }^{29}$ La vinculación de la propia Rocas

28 Bruguera, 2017, 15.

29 Bacardí, 2016, 13. 
con la labor cultural era ingente: realizó trabajos para el Arxiu d'Etnografia i Folklore de Catalunya, para el Arxiu de l'Obra del Cançoner Popular de Catalunya, para el Institut d'Estudis Catalans y fue la corresponsal en la localidad gerundense de Llofriu - de donde era oriunda y donde pasó la mitad de su vida - del proyecto del Diccionari Català-Valencià-Balaear que dirigió Antoni Maria Alcover. ${ }^{30}$ Así, Gràcia Bassa creció en un ambiente de catolicismo, catalanismo y «feminismo burgués» ${ }^{31}$ que consideraba la acción cultural como un propósito positivo para la vida de la mujer, en consonancia con el proyecto político que se afianzaba a comienzos del siglo XX con el liderazgo de la Lliga Regionalista de Catalunya. Bassa llevó a cabo sus actividades culturales en la esfera pública sin descuidar sus obligaciones para con el ámbito familiar, de acuerdo al canon cultural e ideológico que el catalanismo reservaba a la mujer..$^{32}$ Graduada en prestigiosa Escola d'Institutrius i Altres Carreres per a la Dona, fue docente, incursionó en el periodismo cultural en revistas como Feminal o D'aci i d'allà, realizó trabajos de campo como folclorista y ganó prestigio como poetisa en diversas ediciones de los Jocs Florals catalanes. ${ }^{33}$

Al llegar a la Argentina y establecerse en Trenque Lauquen primero y en Buenos Aires desde 1920, Bassa continuó con su vinculación intelectual con Cataluña ${ }^{34}$ pero también, paulatinamente, se integró al ambiente catalanista que despuntaba desde comienzos del siglo y que se afianzó en la Argentina a partir del estallido de la Primera Guerra Mundial, sobre todo desde que Ressorgiment de Buenos Aires pudo difundir de manera sistemática el pensamiento de los «catalanes de América». ${ }^{35} \mathrm{Si}$ bien publicó artículos en otras revistas como Catalunya al Plata, El Correo de Cataluña, Vida Catalana o Catalunya Nova, fue su vinculación con la revista porteña la que le permitió desarrollar su prolongada carrera como periodista. En ese sentido, dos características de la inmigración que llegaba a la joven república americana contribuyeron a moldear esta dedicación. Por un lado, y de manera más general, el espacio de acción del colectivo inmigrante femenino — debido a la saturación de oferta de mano de obra que sufría

30 Vila, 1997, 147 y 149.

31 Palau, 2012, 31; 2013, 35. Bacardí, 2016, 10 y ss.

32 González i Vilalta, 2006, 6

33 Bacardí, 2016, 30. Bassa también se haría un espacio de prestigio como poetisa en los Jocs Florals de Buenos Aires. Bassa de Llorens [Gràcia B. de Llorens], «Discurs presidencial», Ressorgiment, 65, Buenos Aires, 1921, 1035.

34 Bacardí, 2016, 19.

35 Lucci, 2015a. 
el mercado laboral local proveniente de la mayoritaria inmigración masculina $^{36}$ - estaba constreñido a las tareas domésticas o a empleos para los que se requería poca especialización. ${ }^{37}$ En lo que respecta a la colectividad catalana, el sector catalanista, que se mantenía actualizado tanto de las premisas de su ideario cuanto del acontecer político catalán, ${ }^{38}$ reproducía en la comunidad ultramarina las costumbres de la madre patria respecto de la misión de la mujer en la preservación y expansión cultural, sobre todo a partir de su labor de concienciación en el seno de la familia: «La mujer es además uno de los factores primordiales para el resurgir de una raza; su cooperación se hace casi indispensable. Ella inculca a sus hijos el amor a la santa causa; su palabra, llena de dulzura, insufla la convicción a los corazones».$^{39}$ No obstante, había introducido modificaciones paulatinas que descansaban en el contacto con la vida del país de acogida, donde la creciente conflictividad social mostraba un progresivo compromiso femenino en las reivindicaciones sociales y políticas urbanas. ${ }^{40}$ Así, por ejemplo, en el Casal porteño las mujeres no podían ser socias numerarias, ya que solo alcanzaban ese estatus los varones «catalanes e hijos de catalanes». ${ }^{41}$ A partir de 1909, sin embargo, los estatutos de la entidad se modificaron para que las mujeres que quisieran colaborar económicamente con la asociación a título personal pudieran hacerlo. En la acción cultural sí que era evidente la acción femenina, que estaba presente en el Orfeó, el Esbart Infantil, y en la Agrupació Dramàtica. ${ }^{42}$ En el periodismo también comenzaba a notarse la impronta femenina, aunque, como bien lo muestra Ressorgiment, no hay artículos editoriales firmados por mujeres. La presencia femenina era minoritaria en comparación con la cantidad de colaboradores con que contó la publicación a lo largo de su existencia, ${ }^{43}$ pero, aunque sus trabajos estuvieran ligados esencialmente a la literatura y a la difusión del idioma catalán en el exterior, encontramos a Caterina Albert, Emma Alonso da Costa o Emilia y Josefa Bertrán. Ressorgiment también publicó una veintena de artículos de la periodista catalano-chilena Carlota Guteras, quien desarrolló una consistente

36 Sallé Alonso y Van den Eynde, 2009, 16.

37 Núñez Seixas, 2001, 76

38 Lucci, 2014.

39 Muralla, «Optimisme», Ressorgiment, 2, Buenos Aires, 1916, 22.

40 Molyneux, 2009. Graciano, 2010

41 «Estatuto del Casal Català de Buenos Aires», Buenos Aires, 1918, Archivo del Comité Llibertat (ACL), colección particular, sin signatura, art. II, f. 1r.

42 Bacardí, 2009, 14-15. Carbó i Farré, 1995.

43 Lucci, 2010. 
y prestigiosa carrera sobre todo a partir de sus colaboraciones en otra publicación catalanista ultramarina, Germanor de Santiago de Chile. ${ }^{44}$ Así, si tenemos en cuenta la centralidad de la acción cultural en el ideario catalanista, podemos afirmar que la contribución femenina estuvo presente en el panorama del colectivo emigrado y exiliado americano.

Fue en ese contexto en el que Gràcia Bassa comenzó su colaboración sistemática con la revista de Nadal a partir de su columna «Gloses Femenines» en abril de 1917. ${ }^{45}$ Para estos escritos, — que se conocerán definitivamente como «Glosses Femenines» a partir de febrero de 1921 cuando el título adoptó los cánones de la normalización lingüística del catalán llevada a cabo por Pompeu Fabra ${ }^{46}$ — Bassa utilizó el seudónimo de Alidé. Esta firma fue la primera de muchas que utilizaría en Ressorgiment, ya que también se presentaría como Gracieta B. de Llorens, Maria Muntanya o Núria Montseny. ${ }^{47}$ El primer seudónimo tiene reminiscencias griegas, pero sobre todo constituye una referencia directa al poema «La poma escollida», de Josep Carner, uno de los referentes centrales del Noucentisme, la corriente artística que marcó la cultura catalana sobre todo durante la primera década del siglo $\mathrm{XX} .{ }^{48}$ Por lo tanto, la primera aparición de la periodista en el entorno de la prensa étnica porteña sería una carta de presentación integral, desde la propia firma, que la vinculaba a su tarea folclórica previa de preservación cultural y a su vocación literaria.

La entrega inicial de las «Glosses» es breve, pero es el punto de partida medular para comprender desde qué parámetros Bassa buscó integrarse en el incisivo discurso de Ressorgiment, donde, en la misma página en la que se publicaba la presentación de Alidé, se volvía a exponer la necesidad de redoblar los esfuerzos de la «tarea patriótica» cultural del catalanismo americano. ${ }^{49}$ Indica, en primer término, la perspectiva que tendrían los comentarios - las glosas del título- que estarían marcadas por la óptica de una mujer. El plural utilizado revela también, de manera elíptica, que el destinatario ideal es el colectivo femenino, premisa que devenía explícita en el subtítulo, «Saludo a las catalanas de América», y en las primeras líneas del texto: «Vengo a vosotras, mujeres catalanas de América, para iniciar una tanda de conversaciones, animada por la confianza en vuestra benevolencia

44 Corretger, 2005.

45 Bassa de Llorens [Alidé], «Gloses Femenines», Ressorgiment, 9, Buenos Aires, 1917, 137.

46 Utilizaremos esta grafía a lo largo del artículo.

47 Ressorgiment, Buenos Aires, 1916-1960. Bacardí, 2016, 38.

48 Costa Ruibal, 2002.

49 Alemany i Borràs, «Perque ens descatalanitzem?», Ressorgiment, 9, Buenos Aires, 1917, 137. 
hacia mí». Esta conexión buscaba establecer una comunicación «fraternal» con la «mujer catalana ideal», que para Bassa estaba comprometida de manera indisoluble con el cuidado del hogar y la preservación de la cultura: «muchachas en la flor de la vida; educadoras, dulces compañeras, madres, formadoras cabales de los ciudadanos del mañana; exquisitas artistas e intelectuales que estáis de paso o que desgranáis vuestra vida trasplantadas en esta tierra; [me dirijo] tanto a las clarividentes, cuanto a las desvalidas y desorientadas». Como la preparación de futuros ciudadanos de una posible Cataluña independiente también le preocupaba, recordaba y agradecía la presencia de medios como Ressorgiment, «preciado portavoz de nuestra espiritualidad, de toda nuestra vida y del lazo que nos une con la patria lejana» para complementar en el exterior la labor formativa de la mujer. ${ }^{50}$ El texto presenta un aspecto de concordancia entre las «Glosses» con la política editorial de Nadal: la gratitud y el respeto a la Argentina como tierra de acogida y la admiración del director de Ressorgiment por el ejemplo de vida democrática del país americano. ${ }^{51}$ Bassa se sumaba a esta actitud saludando en nombre de sus lectoras a la hospitalidad de «la noble Argentina que quiere nuestro concurso de sentimiento y de acción, aunque nos sepa doloridas, en su alma colectiva». ${ }^{52}$

\section{Los años fructíferos del glosario femenino}

El período fecundo de estas crónicas femeninas es el comprendido entre 1917 y 1925, si bien Bassa, durante los primeros años de la década de 1920, comenzó a colaborar en Ressorgiment con artículos individuales dirigidos a sus lectores en general. La mirada a la vez optimista y melancólica de Alidé se convirtió en la marca distintiva de su discurso. La tristeza de la lejanía y la pulsión por colaborar con los ideales nacionales de Cataluña se amalgamarían de manera inseparable, fruto de su añoranza proactiva respecto de su tierra natal, ${ }^{53}$ y marcarían la inflexión de las «Glosses Femenines». Las «Glosses» señalan también dos rasgos que serán permanentes en los escritos de Bassa. En primer lugar, no se ocuparán de temas de actualidad rigurosa —más ligados a las crónicas

50 Bassa de Llorens [Alidé], «Gloses Femenines», Ressorgiment, 9, Buenos Aires, 1917, 137.

51 Nadal i Mallol, «Unes paraules», Ressorgiment, 1, Buenos Aires, 1916, 1

52 Bassa de Llorens [Alidé], «Gloses Femenines», Ressorgiment, 9, Buenos Aires, 1917, 137.

53 Idem. 
periodísticas - sino que serán reflexiones o análisis sobre temas transversales, comprensibles en contextos de procesos más que en coyunturas del corto plazo. En segundo término, el problema catalán en su relación con el estado español y el catalanismo - tanto como base cultural de la identidad nacional cuanto como pensamiento político legitimador de las reclamaciones nacionales - tendrían siempre preponderancia en el contenido de los artículos o constituirían la perspectiva elegida para las temáticas escogidas en cada oportunidad.

Una de las primeras entregas de las «Glosses Femenines» sentó las bases de su opinión sobre lo que Alidé denominaba el «carácter catalán». En primer lugar, expresaba su incondicional afecto por todos los componentes de «la manera de ser de Cataluña: la amamos en todos sus defectos, que son la sombra que hace aflorar más luminosas y gloriosas sus enormes virtudes; las dos tendencias las consideramos patrimonio de nuestra carnadura humana». Esta identificación incondicional devenía de inmediato en la razón que justificaba su papel en la sociedad catalana y su objetivo como parte de la colectividad catalanista ultramarina: «Trabajemos siempre con voluntad y fe por el enaltecimiento, en todo el mundo, de nuestra dulcísima y amada Cataluña»..$^{54}$

Sin embargo, durante los años del final de la Primera Guerra Mundial y el comienzo del Período de Entreguerras, las «Glosses» acercaron a las lectoras reflexiones sobre temas que desde distintos ángulos enfatizaban el papel creciente de la mujer en la sociedad. En julio de 1917, Bassa se significó en favor del voto femenino. Sin hacer referencia directa al problema catalán y poniéndolo así en una perspectiva más global acorde con la coyuntura de la Gran Guerra, Alidé instaba a las mujeres a dejar de actuar «ligadas a viejos prejuicios» que ya no se sostenían, ya que la posibilidad de alcanzar gobiernos democráticos no podía dejarse exclusivamente en manos de la población masculina, pues consideraba que a veces hacía ejercicio de su derecho al voto de manera indolente, «sin tener conciencia de su importancia y responsabilidad». Esa glosa relacionaba primero el voto con la necesidad de una educación femenina acorde con las obligaciones comunes que conllevaba ejercer «dignamente las eminentes responsabilidades y prerrogativas» que emanaban del ejercicio electoral. Afirmaba también que había que comenzar a trabajar en ese sentido porque la aportación de la mujer era cada vez más indispensable debido a la inclinación natural

54 Bassa de Llorens [Alidé], «Gloses Femenines», Ressorgiment, 13, Buenos Aires, 1917, 201. 
que la definía y que predisponía «su corazón y su pensamiento siempre a un valor ético más elevado». Para Alidé, el rol de la mujer en el entorno familiar no se veía comprometido ya que contemplaba al sufragio como una manera de complementar sus tareas de madre y educadora en la esfera privada y de recuperar «parte de las ideas de paz y serenidad» que la Civilización Occidental parecía haber perdido. ${ }^{55}$ El trágico comienzo del siglo XX, según Alidé, reclamaba de la mujer catalanista dejar atrás «frivolidades y encantamientos» y ampliar su esfera de acción a tareas civiles y sociales que la colocaran «al lado del hombre» en el objetivo de mejorar el derrotero de la humanidad. ${ }^{56}$

Alidé llevaba a las mujeres catalanistas americanas su preocupación por dejar de ver su rol centrado con exclusividad en la esfera familiar. Así, el ideal femenino catalanista reivindicaba sus objetivos, pero adecuado al signo de los tiempos: «En todas sus épocas y en todos sus estamentos, la mujer es madre y maestra». ${ }^{57}$ Pero esta convicción aparecía paulatinamente no como un límite sino como un puente para conectar lo privado con lo público. Otra vez la debacle generada por la Gran Guerra era el sustrato de una reflexión que se proyectaba hacia el porvenir. Para Alidé, las «mujeres rusas que van a la guerra» ${ }^{58}$ protagonizaban un «holocausto» digno de admiración y de misericordia. Pero Alidé pensaba también que estas «voluntarias para el combate» ${ }^{59}$ eran la prueba de que la guerra arrasaba con «todo lo sagrado que hay en el mundo», desintegraba la dualidad recurrente «Madre-Mujer» - que expresaba con mayúsculas para enfatizar su centralidad - y contradecía la percepción femenina del discurso hegemónico del catalanismo. ${ }^{60}$

Pero para finales de la década de 1910, el ejercicio de la actividad política y la formación de futuros ciudadanos no debían ser la vanguardia y la retaguardia de la acción de dos sexualidades disímiles y desconectadas socialmente. Aún desde su rol tradicional, la mujer debía «juntarse en un grito universal $»^{61}$ con el hombre para contribuir a mejorar la convivencia de las naciones: «mucho habrá ganado la humanidad y no serán tan posibles los efectos graves de equivocación de unos cuantos que, no siempre sabios

55 Idem.

56 Bassa de Llorens [Alidé], «Gloses Femenines», Ressorgiment, 12, Buenos Aires, 1917, 183.

57 Idem.

58 Bassa de Llorens [Alidé], «Gloses Femenines», Ressorgiment, 15, Buenos Aires, 1917, 239.

59 Huguet, 2019, 48.

60 Bassa de Llorens [Alidé], «Gloses Femenines», Ressorgiment, 15, Buenos Aires, 1917, 239

61 Bassa de Llorens [Alidé], «Gloses Femenines», Ressorgiment, 29, Buenos Aires, 1918, 459. 
y dignos, rigen eminentemente a los pueblos. No serán posibles las vergonzosas guerras, con su reguero de iniquidades y de víctimas completamente ajenas e inocentes». ${ }^{62}$

La necesidad de mejorar el acceso de la mujer a la educación — arraigada en los cánones culturales y familiares de Bassa- era un tema recurrente que se revelaba, por ejemplo, en la difusión de la personalidad y actividad de aquellas mujeres que se ganaban un sitio en el ámbito científico. Siempre preocupada por dar a conocer los logros femeninos en Cataluña, informaba de los progresos de intelectuales y empresarias como Concepció Ferran, quien se había formado en química biológica en el Instituto Pasteur francés y desempeñaba un cargo de directora en la empresa Indústries Químiques de Barcelona ${ }^{63}$ Pero también difundía casos como el de la Premio Nobel Marie Curie quien, según Alidé afirmaba en 1921, no solo había «acompañado» a su esposo en su trabajo teórico, sino que ella misma era capaz de «abrir nuevos e inmensos horizontes en la medicina y en todo el reino científico». ${ }^{64}$

Así, la educación fue un tema cultural vinculado a la política durante la década de 1920, la última en la que las «Glosses» se publicarían regularmente. Por ende, para Alidé era central tomar consciencia tanto de las carencias educativas de la mujer cuanto de las consecuencias que esta falta de preparación como maestras y como discípulas tenía para la preservación de la identidad nacional y, por tanto, para el devenir político catalán. Una de las urgencias de Alidé — sumándose desde América, igual que Ressorgiment, ${ }^{65}$ a la prédica de la Associació Protectora de l'Ensenyança Catalana ${ }^{66}$ — era la implantación de una educación en lengua catalana y a partir de contenidos preparados por las instituciones de la Mancomunitat de Catalunya:

¡Seguramente está aún lejano ese día en que la mujer esté capacitada para ocupar espacios que demandan una cultura superior que no posee todavía! [...] Un aspecto básico de la cultura de los pueblos reside en la escuela primaria. Desgraciadamente, la nuestra está en manos del opresor. Mientras las generaciones catalanas estén sujetas desde la infancia a un sistema de enseñanza atrasado y carente de amor, no es fácil que la progresión cultural de la mujer catalana llegue a ser una realidad. ${ }^{67}$

62 Bassa de Llorens [Alidé], «Gloses Femenines», Ressorgiment, 21, Buenos Aires, 1918, 336.

63 Bassa de Llorens [Alidé], «Glosses Femenines», Ressorgiment, 73, Buenos Aires, 1922, 1173.

64 Bassa de Llorens [Alidé], «Gloses Femenines», Ressorgiment, 59, Buenos Aires, 1921, 935.

65 Lucci, 2015b.

66 Gonzàlez Agàpito, 2002.

67 Bassa de Llorens [Alidé], «Glosses Femenines», Ressorgiment, 68, Buenos Aires, 1922, 1082. 
Otro tema central de las «Glosses» era el idioma. La difusión del catalán como lengua culta y como instrumento de integración de la mujer en la esfera pública era la razón por la cual informaba sobre las nuevas publicaciones y actividades de «geniales figuras femeninas representantes del arte» de su tierra, ${ }^{68}$ como Víctor Català (seudónimo de Caterina Albert), Clementina Arderiu o María Barrientos ${ }^{69}$ Pero, en este tema, la preocupación de Alidé estaba marcada por el entorno migratorio femenino. Como maestra y escritora vinculada al catalanismo, consideraba que la vitalidad de la lengua catalana era fundamental para la reproducción de la identidad nacional en el exterior. El entorno privado había moldeado la imagen de la mujer catalanista como figura «protectora». ${ }^{70}$ También en el contexto de la experiencia migratoria, esa función le permitió comenzar a operar en la esfera pública, donde descolló como elemento de cohesión identitaria a partir de la difusión, expansión y reproducción cultural. En este sentido, la concordancia ideológica con Ressorgiment era plena. Para Nadal, los catalanes debían utilizar «siempre y en todo el mundo» la lengua materna para proclamar y reproducir la «supervivencia de una patria». ${ }^{71}$

Así, en 1922 Alidé afirmaba que el compromiso de la mujer catalanista ultramarina con su idioma era ontológico: «[debemos] colaborar, cada una a su manera y en la forma más pura y más bella que albergue nuestra voluntad, en la obra de reconstrucción espiritual de nuestra patria». En este punto, el discurso de Alidé cobraba fuerza y se alejaba del tono afable y cercano que caracterizaba a las «Glosses» para dejar claro su mensaje y llegar de manera incisiva a las lectoras: no había excusas para olvidar el idioma catalán porque en él, como expresión secular de la propia cultura, residía la «espiritualidad» que reproducía la identidad. ${ }^{72}$ El texto renegaba con contundencia que fuera debilidad o falta de adaptación al nuevo entorno el hecho de que la mujer lo hablara en el ámbito privado; si acaso, constituía un síntoma de habilidad femenina, que permitía utilizar indistintamente diversos idiomas y no decantarse por el de la tierra de acogida. Para Alidé, un síntoma de flaqueza era renunciar al idioma materno:

68 Bassa de Llorens [Alidé], «Glosses Femenines», Ressorgiment, 73, Buenos Aires, 1922, 1173.

69 Idem. Bassa de Llorens [Alidé], «Glosses Femenines», Ressorgiment, 82, Buenos Aires, $1923,1308$.

70 Casas, 2013, 156

71 Nadal i Mallol, «L'agulló», Ressorgiment, 78, Buenos Aires, 1923a, 1235.

72 Bassa de Llorens [Alidé], «Glosses Femenines», Ressorgiment, 70, Buenos Aires, 1922, 1114. 
La debilidad consiste en que adoptemos el nuevo lenguaje para hablar con nuestros hijos. Eso sí es debilidad manifiesta. Lo es tanto si no sabemos imponernos al medio que nos rodea cuanto si —en caso de considerarlo necesario- nos integramos en él. Y esa debilidad va acompañada de gran ignorancia si creemos que nuestros hijos serán más valiosos si utilizan el idioma de adopción, porque no comprendemos el gran valor del idioma nativo para cada uno, sea el que sea. ${ }^{73}$

En la distancia, por lo tanto, la reproducción del idioma en el ámbito familiar consolidaba las funciones de madre y de maestra que para Bassa definían a la mujer catalanista contemporánea, porque la apartaban de las verdaderas actitudes de debilidad: todas aquellas que, al alejar a los catalanes de su lengua, los llevaba a «atenuar las modalidades» que modelaban su identidad. ${ }^{74}$ En esta tarea cultural tan importante, la mujer debía estar segura de su cometido y de sus capacidades, sobre todo en la coyuntura de Entreguerras, en donde las reclamaciones de autonomía integral catalanas buscaban sumarse a las que hacían oír otras naciones sin estado europeas en los foros diplomáticos internacionales como la Sociedad de Naciones. ${ }^{75}$ La glosa rechazaba con contundencia que la reproducción de la lengua catalana despertara desdén o, lo que es peor, promoviera la autoconmiseración: «Si nosotras no reafirmamos nuestra voluntad honrando en lo que podamos todas las manifestaciones de espiritualidad de Cataluña [...] no queremos llamarnos catalanas porque, menospreciando esta dignidad al no ser entusiastas de nuestra causa en esta hora y en todo momento, no lo merecemos». ${ }^{76}$

\section{Radicalización femenina}

Las últimas «Glosses» de Alidé corresponden a la primera mitad de la década de 1920. Durante ese período abandonaron paulatinamente su regularidad, pero, como contrapeso, reforzaron el tono con que instaban a la mujer catalanista a estar al tanto de la situación política catalana y a comprender la centralidad de su militancia. Esta radicalización no era fortuita. Para la política catalana, esos años marcaron la etapa de eclosión de las reclamaciones de autonomía. El período de Entreguerras dejaba su huella

73 Idem.

74 Idem.

75 Núñez Seixas, 2010.

76 Bassa de Llorens [Alidé], «Glosses Femenines», Ressorgiment, 70, Buenos Aires, 1922, 1114. 
en la política hispánica por partida doble. Por un lado, la radicalización del espectro político catalán estaba propiciada por las reivindicaciones de las pequeñas naciones sin estado que habían formado parte hasta 1918 de los llamados Imperios Centrales y que se convertían en piezas medulares de la geopolítica europea. Por el otro, estaba el conflictivo escenario de la Restauración española, que con la dictadura de Primo de Rivera sumaba la debacle institucional al auge de las derechas y a la crisis socioeconómica que sumían a España en dificultades cada vez más profundas.

Esta inestabilidad local y regional otorgó un espacio ideológico favorable al proyecto de Francesc Macià. Plasmado en el movimiento Estat Català, su pensamiento tendría una aceptación creciente durante los años siguientes no solo en Cataluña, sino también entre la colectividad catalanista ultramarina en general y argentina en especial. Así, en 1922 se fundaron casi de manera simultánea dos asociaciones que tenían como objetivos exclusivos la militancia política. El primero de ellos en Cuba, en La Habana, el Club Separatista Català n. ${ }^{\circ} 1 .^{77}$ En el mismo año, Pere Seras, socio y directivo del Casal porteño fundó el Comité Llibertat, ${ }^{78}$ la segunda entidad separatista de América que se creó con expresos objetivos políticos: «trabajar, al máximo de nuestras posibilidades, para liberar a nuestra patria, desde tanto tiempo oprimida». ${ }^{79}$ Desde 1924 , el Comité apoyaba explícitamente a Macià y, con el paso de los meses, centralizaría buena parte de la militancia separatista americana. ${ }^{80}$ Ressorgiment, que como siempre colaboraba desde el campo intelectual con la difusión de las novedades políticas, opinaba que la tarea cultural y política desde el exterior no era suficiente ayuda y que los «catalanes de América» debían integrarse de manera más efectiva si las reclamaciones catalanas sobrepasaban el ámbito político y alcanzaban la esfera castrense: «¿Creéis, amigos, que será suficiente seguir con el corazón ansioso las nuevas que de allá provengan, o creéis que es necesario convertirnos también en elementos activos? $\gg^{81}$

Por lo pronto, la ayuda económica a Macià se sumó a la tarea cultural: el Empréstito «Pau Claris», por un valor total de nueve millones de pesetas, que en abril de 1925 había puesto en circulación Estat Català para

77 Ruiz García, 2015, 375.

78 «Manifiesto del Comité Llibertat de Buenos Aires», Buenos Aires, 1932, ACL, sin signatura.

79 Carta de propaganda del Comité Llibertat de Buenos Aires, Buenos Aires, febrero de 1925 ACL, sin signatura, f. 1r.

80 Carta del Comité Llibertat a la Associació Protectora de l'Ensenyança Catalana, Buenos Aires, 9 de abril de 1924, ACL, sin signatura, f. 1r.

81 Nadal i Mallol, «Notes d'orientació nacionalista», Ressorgiment, 78, Buenos Aires, 1923b, 1235. 
- aprovechando el escenario dictatorial riverista - organizar la operación militar de Prats de Molló y liberar a Cataluña, recibió un sostenido apoyo ultramarino. ${ }^{82}$ Así, durante esos años, las asociaciones catalanistas habían perfeccionado prácticas sociales y culturales a nivel local, pero también habían podido desarrollar una acción mancomunada sostenida, un objetivo que Ressorgiment había difundido, junto a otras publicaciones ultramarinas, entre la colectividad afecta al catalanismo, a partir de entidades de alcance panamericano como el Comité d'Acció Catalana de Sud Amèrica. ${ }^{83}$

A largo de los años, la adhesión de Bassa a esta posición era palmaria en las «Glosses», que procuraban que la mujer fuera consciente de la nueva etapa de «afirmación nacionalista» en Cataluña. En ese contexto, fortalecían con su discurso el tradicional papel femenino en la sociedad, aún desde el exterior: «Adalides de cultura y de civilidad, son muchas las damas de nuestra Nación que la enaltecen y que estimulan a todos para colaborar en el ansiado objetivo de nuestra libertad» ${ }^{84}$ Para ello, ejemplos paradigmáticos como el de la poetisa y activista Agnès Armengol — quien en 1903 había donado una senyera al partido Unió Catalanista, producto de su propia iniciativa de propugnar una suscripción con ese fin para incentivar la participación femenina en el movimiento catalanista ${ }^{85}$ - o la lectura de textos que orientaran a la mujer en su compromiso patriótico - como D'espiritualitat Femenina, de Jaume Bofill i Matas ${ }^{86}$ _, eran centrales para colaborar con más convicción y para lograr una preparación adecuada que les permitiera incrementar sus responsabilidades públicas. ${ }^{87}$

En el contexto de efervescencia del catalanismo americano, las «Glosses Femenines» buscaban concienciar a la mujer para que continuara su labor de apoyo al catalanismo integrándose a las reivindicaciones de autonomía de ese momento. Para ello, recalcaban las características y la necesidad de su aportación tradicional: «Donde sea que se realicen actos patrióticos, el corazón de la mujer catalana está siempre latente, de manera más o menos ostensible, porque ella siente que esa actitud no tiene nada de vano ni de foráneo». ${ }^{88}$ Alidé volvía a recalcar así lo que consideraba distintivo del carácter de la mujer catalana, quien desde la «sencillez» de su persona y de su

82 Faura i Homedes, 1991.

83 Lucci, 2015a.

84 Bassa de Llorens [Alidé], «Glosses Femenines», Ressorgiment, 78, Buenos Aires, 1923, 1236

85 Canyà, 1993, 56.

86 Bofill i Matas, 1916.

87 Bassa de Llorens [Alidé], «Glosses Femenines», Ressorgiment, 78, Buenos Aires, 1923, 1236.

88 Ibidem, 1235. 
ámbito natural de acción — la familia — actuaba con modestia y constancia al lado del hombre.$^{89}$ En el centésimo número de Ressorgiment, en noviembre de 1924, las «Glosses» afirmaban que la acción femenina descansaba en la reflexión sobre las gestas del pasado, ya que serenaban el ánimo y eran el ejemplo necesario de «inmortalidad» que proveían de inspiración a las «tareas de combate y reafirmación patrióticas». ${ }^{90}$ Pero serenidad o delicadeza no debían confundirse con debilidad. El derrotero político podía generar dudas aún entre «los catalanes de corazón»; pero no solo la vacilación, tampoco el celo patriótico irreflexivo era recomendable, pues podía acarrear efectos contraproducentes. El lema que debía unir a la comunidad catalanista a ambos lados del Atlántico era para ella simple: «Creo, espero y amo». Esa consigna uniría a los dirigentes decididos, a los seguidores convencidos y a los indecisos en una militancia efectiva. La unión en la confianza era para Alidé el camino a seguir: «Nuestra aspiración patriótica que clama por la victoria de la Patria vendrá porque responderá al anhelo de nuestros corazones, que piden por ella. De la misma manera, se alejaría si nos viera asustados o dándole la espalda». ${ }^{91}$

Para septiembre de 1925, Alidé afirmaba que la aportación femenina necesitaba nutrir su discreción con otros rasgos de carácter más intensos: «La mujer catalana no es nada guerrera. Pero tal vez sería bueno que lo fuera». ${ }^{92}$ Colaborar en el ámbito de la cultura y sostener con recursos propios el Empréstito «Pau Claris» podía no ser suficiente. Si lo que consideraba como el «doloroso problema de Cataluña» lo requería, la mujer catalanista no debía luchar, pero sí, desde la esfera privada, reforzar su propio carácter para educar en valentía a la juventud:

Para eso sería bueno que las catalanas fuéramos de temperamento guerrero, porque enseñaríamos a nuestros niños, para que se les hiciera natural y después fueran ellos guerreros, sin pesar ni desconcierto como ahora, que son educados con los preceptos de las miras más modernas y elevadas, y el mundo no se lo merece. ${ }^{93}$

La falta de regularidad de las «Glosses», por lo tanto, no solo no implicó un desinterés de Gràcia Bassa por la cuestión catalana, sino que puede considerarse un síntoma inequívoco de su madurez ideológica. Efectivamente,

89 Bassa de Llorens [Alidé], «Glosses Femenines», Ressorgiment, 110, Buenos Aires, 1925, 1788.

90 Bassa de Llorens [Alidé], «Glosses Femenines», Ressorgiment, 100, Buenos Aires, 1924, 1631.

91 Bassa de Llorens [Alidé], «Glosses Femenines», Ressorgiment, 101, Buenos Aires, 1924, 1640.

92 Bassa de Llorens [Alidé], «Glosses Femenines», Ressorgiment, 110, Buenos Aires, 1925, 1788.

93 Idem. 
hacia finales de la década de 1920, sus intereses hacia la opinión pública sobrepasaron las audiencias femeninas para intentar llegar, desde la revista vocera de los «catalanes de América» a los lectores de la publicación desde la visión «amable y serena» ${ }^{94}$ pero contundente de una mujer catalanista: «Particularmente nosotras, mujeres, en el seno de la familia y en la sociedad y en el ambiente en el que vivimos, tenemos cosas que decir; nos las inspirará solamente nuestra dignidad de ser catalanas. ¡Fuera cobardía! Decidlo en voz alta, porque así debe ser». ${ }^{55}$ Su propio entorno familiar era afín al catalanismo: el matrimonio Llorens mantenía una relación cercana con Macià que ha quedado registrada en la correspondencia que el político le enviaba a su esposa, Eugènia Lamarca, desde Buenos Aires ${ }^{96} \mathrm{El}$ apoyo al proyecto del líder catalán era explícito al punto tal que, en 1926, cuando él y su secretario Ventura Gassol tuvieron serios inconvenientes para lograr la residencia legal en Argentina, luego de los sucesos de Prats de Molló, la pareja los mantuvo ocultos en su casa familiar en Buenos Aires hasta que regularizaron su situación en el proceso que se llevó a cabo ante la Corte Suprema de Justicia argentina. ${ }^{97}$ Paulatinamente, los artículos periodísticos de Gràcia Bassa en Ressorgiment — que durante esa década habían coexistido con las «Glosses»—- se convirtieron en su medio de expresión periodístico por excelencia y, durante 1930 y 1940, continuaron con la prédica catalanista obviando los análisis coyunturales de sucesos específicos pero sirviéndose de la cultura, la historia, la literatura y la política para expresar su visión femenina de la problemática peninsular que definiera la convivencia entre catalanes y españoles: «Son extranjeros para nosotros, y los únicos lazos que podemos, con dignidad, tener con ellos son los de vecindad más o menos forzosos. Si alguna cosa puede unirnos políticamente es la aquiescencia recíproca que genere una felicidad y convivencia mutua, de carácter internacional.» ${ }^{98}$

94 Idem

95 Bassa de Llorens, «Fragments. Conferència al Centre Català de Cultura de La Plata», Ressorgiment, 95, Buenos Aires, 1924, 1520.

96 Carta de Francesc Macià desde Buenos Aires a Eugènia Lamarca, Buenos Aires, 28 de junio de 1928, Arxiu Nacional de Catalunya (ANC), Francesc Macià i Llusà, «Correspondència de Macià, F. a Lamarca, E. (1907-1930)», ANC1-264-T-1399, f. 2r. Lucci, 2011.

97 Este proceso fue llevado adelante por el letrado Alfredo Palacios — quien se convertiría en el primer legislador socialista de América al ocupar una banca por el Partido Socialista en la Cámara de Diputados de la Argentina en 1904- y el abogado Claudio Caminos. Carta de Francesc Macià desde Montevideo al Comité Llibertat, Montevideo, 19 de febrero de 1928, ANC, Francesc Macià i Llusà, «Cartes d'en Macià al Comitè Llibertat de Buenos Aires», ANC1-264-T-1806, f. 1r. Palacios y Caminos, 1929.

98 Bassa de Llorens [Núria Montseny, seud.], «Vers la nostra llibertat», Ressorgiment, 188, Buenos Aires, 1932, 3033. 


\section{Conclusiones}

Las «Glosses Femenines» nos acercaron a aspectos de la producción periodística de Gràcia Bassa que no habían sido estudiados en profundidad. En una perspectiva más general, nos han permitido situar culturalmente a Bassa en el contexto de dos influencias vitales que consideramos centrales para su biografía intelectual: por un lado, el catalanismo, por el otro, la emigración. El primero nos ha permitido comprobar la vinculación entre su trabajo como folclorista, su producción poética y periodística en Cataluña y los textos que publicó en la Argentina. Esta concatenación ideológica - centrada esta vez en la perspectiva de género - ha abierto una nueva vía de análisis sobre la manera en que el catalanismo cristalizó en el pensamiento y la acción de los «catalanes de América», favorecido por el fluido contacto entre el Nuevo Mundo y España durante el período de emigración masiva. Efectivamente, las «Glosses» nos acercaron por primera vez a la perspectiva femenina del catalanismo separatista radical de ultramar. A través de los textos que firmó como Alidé, establecimos la manera en que Gràcia Bassa construyó su particular forma de colaborar en la acción cultural — tan importante para el pensamiento catalanista- durante las primeras décadas del siglo XX. La experiencia migratoria, por su parte, nos ha permitido estudiar la manera en que la sociedad de acogida influyó en su cosmovisión. Los artículos de Alidé — vinculados al objetivo de reforzar la cohesión identitaria entre las mujeres emigrantes catalanistas - coadyuvaron a potenciar su percepción de la importancia de la función de la mujer como productora de cultura. En ese sentido, hemos podido rastrear la impronta de la vida política argentina en los artículos estudiados y la manera en que Bassa reconocía la influencia que los parámetros de convivencia y libertad de la sociedad de acogida tenían en la labor de expansión cultural y social del catalanismo. Por último, hemos reforzado la importancia de Ressorgiment como corpus documental para entender las estrategias y tácticas de acción de los «catalanes de América» hasta por lo menos el comienzo de la Guerra Civil española.

De manera más concreta, nuestro análisis de las «Glosses Femenines» nos ha permitido efectuar una contribución original a los estudios sobre folclorismo catalanista y sobre el papel que la mujer desempeñó en la preservación y expansión de la cultura catalana durante las primeras décadas del siglo pasado. En primer lugar, hemos podido reforzar con nuevos sujetos históricos y fuentes inéditas que los entornos de acción catalanista en Cataluña y en América no aparecían disociados, sino todo lo contrario. 
La permanencia de Alidé en las páginas de Ressorgiment permite comprobar la vinculación del separatismo americano con el entorno peninsular del que obtuvo y construyó su marco teórico, pues permitió a la periodista retomar su actividad intelectual con comodidad en la prensa catalanista porteña. En segundo lugar, resaltan la percepción del feminismo de Bassa, que aparece totalmente consustanciado con el catalanismo: Alidé habló como mujer catalanista a la mujer catalanista y su visión feminista, por lo tanto, estaba constreñida a la percepción de la mujer, de su función y de sus aspiraciones en el contexto del problema catalán. En tercer término, y ligado indisolublemente a lo anterior, las «Glosses» nos han desvelado la particular conformación de la cosmovisión de Bassa respecto del rol de la mujer en general y de su función en particular. En esta etapa hemos identificado un rasgo conservador en el pensamiento de Gràcia Bassa, quien hasta mediados de la década de 1920 - y a pesar de exponer los beneficios de que la mujer expandiera sus inquietudes a la esfera pública — aparecía todavía anclada en pautas de comportamiento tradicionales, que reivindicaban la prudencia y la modestia de carácter y vinculaban la hegemonía de la acción femenina a las labores de madre y educadora, sobre todo en el entorno familiar. Sin embargo, su propio desarrollo personal — ligado de manera cada vez más evidente a la tarea periodística - también permite establecer una cuarta característica de las «Glosses»: una apertura paulatina hacia posiciones de vanguardia cuyas raíces hemos podido identificar no solo en su acervo tradicional sino también en la perspectiva que le otorgó su arraigo definitivo fuera de Cataluña. Así, hemos encontrado en Alidé una pluma en la que esos rasgos conservadores conviven con su atención a las opciones vitales femeninas fuera del entorno privado, lo que la llevó a reflexionar sobre las aportaciones de la mujer a la sociedad moderna, sobre la necesidad de que mejorara radicalmente su educación y sobre la pertinencia de que lograra ejercer el derecho al voto. Hemos comprobado, en ese sentido, que la adhesión de Bassa - y de su entorno familiar e intelectual — al proyecto de Estat Català marcó una insoslayable reafirmación de este discurso más proactivo en el que la mujer catalanista, aún sin renunciar a su rol tradicional, debía introducir en su carácter rasgos menos contemplativos y congeniar su vocación maternal con un espacio activo en la acción política catalanista ultramarina.

De esta manera, nos hemos acercado a la presencia femenina en la acción cultural catalanista porteña. Esta óptica, poco trabajada, nos permite reflexionar sobre la impronta de una aportación que, aunque minoritaria en número, merece una atención continuada para estudiar sus características 
específicas y su impacto en el colectivo catalanista ultramarino en general y argentino en particular. Será necesario, para ello, emprender estudios de género y de historia sociocultural comparada desde los cuales continuar con el análisis del discurso de Gràcia Bassa en América para explorar más a fondo su pensamiento y evolución pero, sobre todo, para integrarlo al del resto de voces de mujeres emigrantes y exiliadas que buscaron y encontraron un espacio en sus países de acogida desde el cual reflexionar sobre su experiencia migratoria.

Recibido, 27 de noviembre de 2019 Segunda versión, 26 de febrero de 2020 Aceptado, 23 de marzo de 2020

\section{Referencias bibliográficas}

Alemany i Borràs, Joan B., «Perque ens descatalanitzem?», Ressorgiment, 9, Buenos Aires, 1917, 137.

Archilés, Ferran, «Introducción: más allá de una dicotomía», en Archilés, Ferran (ed.), No solo cívica. Nación y nacionalismo cultural español, Valencia, Tirant lo Blanch, 2018, 9-24.

Bacardí, Montserrat, Gràcia Bassa, poeta, periodista i traductora, Palafrugell, Institut de Mitjans de Comunicació Pública de Palafrugell, 2016.

Bacardí, Montserrat, Records de Fivaller Seras, Barcelona, Pagés, 2009.

Bassa de Llorens, Gràcia [Alidé, seud.], «Gloses Femenines», Ressorgiment, Buenos Aires, 1917-1921.

Bassa de Llorens, Gràcia [Alidé, seud.], «Glosses Femenines», Ressorgiment, Buenos Aires, 1921-1925.

Bassa de Llorens, Gràcia [Gràcia B. de Llorens], «Discurs presidencial», Ressorgiment, 65, Buenos Aires, 1921, 1035.

Bassa de Llorens, Gràcia [Gràcia B. de Llorens], «Fragments. Conferència al Centre Català de Cultura de La Plata», Ressorgiment, 95, Buenos Aires, 1924, 1520 .

Bassa de Llorens, Gràcia [Gràcia B. de Llorens], «El tema planyívol», Ressorgiment, 365, Buenos Aires, 1946, 5932.

Bassa de Llorens, Gràcia [Gràcia B. de Llorens], «Els fets gloriosos», Ressorgiment, 490, Buenos Aires, 1957, 7891.

Bassa de Llorens, Gràcia [Núria Montseny, seud.], «Vers la nostra llibertat», Ressorgiment, 188, Buenos Aires, 1932, 3033.

Bofill i Matas, Jaume, D'espiritualitat femenina. Conferencia II de la serie organitzada por la M. Iltre. Junta de Dames de Barcelona, Barcelona, Bloud y Gay Editors, 1916. 
Bruguera, Mónica, «Género y subjetividad en la España del siglo xix. (Un diálogo entre la historia y la literatura)», Espacio, Tiempo y Forma, Serie V Historia Contemporánea, 15, Madrid, 2017, 15-19.

Canyà, Llucieta, «El periodismo i les dones de la Renaixença. Conferència a Ràdio Associació (Dia 22 de juliol)», Annals del periodisme Català, 1, Madrid, 1933, 49-60.

Carbó i Farré, Josep, El Casal Català de Buenos Aires. Text de la conferència pronunciada el dia 9 de maig de 1970 a l'Obra Cultural Catalana, Buenos Aires, Index, 1995.

Casas, Saúl Luis, «Militancia republicana, identidad nacional y sociabilidad comunitaria de los catalanes en la Argentina (1920-1945)», tesis de posgrado dirigida por A. Fernández, Universidad Nacional de La Plata, Facultad de Humanidades y Ciencias de la Educación, 2013. Disponible en www.memo ria.fahce.unlp.edu.ar/tesis/te.1047/te.1047.pdf. [Consultado: 03-08-2019].

Corretger, Montserrat, «Autobiografia intel-lectual de Carlota Guteras a partir de les relacions epistolars amb Cristòfor de Domènech (1924-1927) i Joan Estelrich (1923)», en Cortés Ort, Carles et al. (eds.), Epístola i literatura. Epistolaris, la carta: estrategia literaria, Paiporta, Editorial Denes, 2005, 155-181.

Costa Ruibal, Oscar, L'imaginari imperial: el Noucentisme català i la política internacional, Barcelona, Editorial Alpha, 2002.

Faura i Homedes, Ricard, El complot de Prats de Molló, Barcelona, El Llamp, 1991.

Fernández, Alejandro, «Persistencias y rupturas en la identidad española de los intelectuales catalanes de Buenos Aires (1850-1950)», Anuario IEHS-Instituto de Estudios Histórico-Sociales, 31, Tandil, 2016, 85-102.

Gonzàlez Agàpito, Josep, Tradició i renovació pedagògica, 1898-1939: història de l'educació: Catalunya, Illes Balears, País Valencià, Barcelona, L'Abadia de Montserrat, 2002.

González i Vilalta, Arnau, La irrupció de la dona en el catalanisme, 1931-1936, Barcelona, L'Abadia de Montserrat, 2006.

Graciano, Osvaldo, «El Partido Socialista de Argentina: su trayectoria histórica y sus desafíos políticos en las primeras décadas del siglo XX», A Contra Corriente, 3, Buenos Aires, 2010, 1-37.

Huguet, Montserrat, «Mujeres patrióticas en la Rusia de 1917», Revista de historiografía, 31, Madrid, 2019, 37-64.

Jofre Cabello, Ana, Así emigraron los Baleares a la Argentina, Palma, Conselleria de Presidència del Govern Balear, 1997.

Llopart, Dolors y Ros, Roser, «Dona i folklore. Apunts per a l'estudi del folklore compilat per dones a cavall de finals del segle xix i les primeries del segle XX», en Temporal, Josep y Villalba, Laura (eds.), La recerca folclórica: persones $i$ institucions, Alicante, Industrias Gráficas Alicante, 2012, 43-54.

Louis, Anja, «La identidad feminista en la obra de Carmen de Burgos», Estudios románicos, 27, Murcia, 2018, 33-48. 
Lucci, Marcela, «La Colectividad catalana en Buenos Aires en el siglo XX: una visión a través de los catalanes de los "catalanes de América". El activismo patriótico de los catalanes de América de Buenos Aires: desde 1916 hasta el final del Casal Català», tesis doctoral dirigida por B. de Riquer i Permanyer, Universitat Autònoma de Barcelona, Departamento de Historia Moderna y Contemporánea, 2010. Disponible en: http://hdl.handle.net/10803/4820. [Consultado: 16-7-2019].

Lucci, Marcela, «Francesc Macià en la prensa argentina: el asociacionismo catalanista porteño y la gestión del apoyo a la causa del independentismo catalán en Crítica», Estudios de Historia de España, 14, Buenos Aires, 2011, 185-212.

Lucci, Marcela, «La Revista Ressorgiment i el separatisme d'ultramar: aportacions indispensables per a l'estudi del catalanisme a Amèrica», Recerques: història, economia, cultura, 63, Valencia, 2014, 33 - 59.

Lucci, Marcela, «La Globalización del catalanismo de entreguerras: corporaciones americanas para la gestión internacional del activismo separatista», en Grageda Bustamante, Aarón (coord.), Intercambios, actores, enfoques: pasajes de la historia latinoamericana en una perspectiva global, Hermosillo, Universidad de Sonora, 2015a, 83-100.

Lucci, Marcela, «El Papel de la educación en el proyecto político del catalanismo ultramarino: la revista Ressorgiment de Buenos Aires durante la primera mitad del siglo XX», Amnis. Revue de civilisation contemporaine. Europes/ Amériques, 14, Marsella, 2015b. Disponible en https://journals.openedition. org/amnis/2604 [Consultado: 12-04-2019].

Lucci, Marcela, «Nuevos Documentos para la historia sociocultural española. Perspectivas renovadoras para el estudio del asociacionismo catalán en Argentina durante la primera mitad del siglo XX», RiMe, Revista Online del Istituto di Storia dell'Europa Mediterranea, 17, 1, Cagliari, 2016, 35-61. Disponible en: https://doi.org/10.7410/1205 [Consultado: 12-04-2019].

Mignone, Aníbal Marcelo, «El sistema ferroviario de la República Argentina», Resúmenes. Revista Geográfica Digital. IGUNNE [Instituto Geográfico de la Universidad Nacional del Nordeste], 19, Resistencia (Argentina), 2013. Disponible en: https://hum.unne.edu.ar/revistas/geoweb/Geo19/archivos/ mignone13.pdf [Consultado: 27-04-2019].

Miralles, Xavier Andreu, «El género de las naciones. Un balance y cuatro propuestas», Ayer, 106, Madrid, 2017, 21-46.

Molyneux, Maxine, Movimientos de mujeres en América Latina: estudio teórico comparado, Valencia, Universitat de València, 2003.

Muralla, «Optimisme», Ressorgiment, 2, Buenos Aires, 1916, 22.

Nadal i Mallol, Hipòlit, «Unes paraules», Ressorgiment,1, Buenos Aires, 1916, 1.

Nadal i Mallol, Hipòlit, «L'agulló», Ressorgiment, 78, Buenos Aires, 1923a, 1235.

Nadal i Mallol, Hipòlit, «Notes d'orientació nacionalista», Ressorgiment, 78, Buenos Aires, 1923b, 1235. 
Núñez Seixas, Xosé Manoel, Internacionalitzant el nacionalisme: El catalanisme polític la qüestió de les minories nacionals a Europa (1914-1936), Valencia, Universitat de València, 2010.

Núñez Seixas, Xosé Manoel, La Galicia austral: la inmigración gallega en la Argentina, Buenos Aires, Biblos, 2001.

Ortuño Martínez, Bárbara, «La imagen de la(s) madre(s). Construcciones identitarias en los procesos migratorios vividos durante la infancia», en Ibarra Aguirregabiria, Alejandra et al. (coords.), No es país para jóvenes, VitoriaGasteiz, Instituto Valentín de Foronda, 2012. Disponible en: https://dialnet. unirioja.es/servlet/articulo?codigo=4715138 [Consultado: 12-04-2019].

Palacios, Alfredo y Caminos, Claudio, Derecho de Asilo. (Caso Macià Gassol), Buenos Aires, Editorial Claridad, 1929.

Palau, Montserrat, «Les aportacions al feminisme de les folkloristes Maria Gràcia Bassa i Maria Baldó», en Temporal, Josep y Villalba, Laura (eds.), La recerca folclórica: persones $i$ institucions, Alicante, Industrias Gráficas Alicante, 2012, 13-26.

Palau, Montserrat, «L'accés de les dones catalanes al món cultural i als estudis de folklore», en Llopart, Dolors y Ros, Roser (eds.), Dona i folklore, Barcelona, Tantágora, 2013, 29-36.

Riquer i Permanyer, Borja de, «Modernitat i pluralitat, dos elements bàsics per a entendre i analitzar el catalanisme», en Anguera, Pere et al., El catalanisme conservador, Girona, Centre d'estudis socials de Girona, 1996, 7-23.

Ruiz García, Sergio, «El asociacionismo español en Cuba: un encuentro de identidades: el caso catalán (1840-1940)», tesis doctoral dirigida por Consuelo Naranjo Orovio y Manuel Herrero Sánchez, Universidad Pablo de Olavide, Sevilla, Departamento de Geografía, Historia y Filosofía, 2015. Disponible en: http://hdl.handle.net/10433/2146 [Consultado: 27-08-2019].

Sallé Alonso, María Ángeles y Van den Eynde, Ángeles (eds.), La emigración española en América: Historias y lecciones para el futuro, Madrid, Fundación Directa, 2009.

Servén Díez, Carmen, «Mujeres y prensa: la página femenina de El Sol (19171936)», en Libro de Actas del I Congreso Internacional de Comunicación y Género, Sevilla, Facultad de Comunicación de la Universidad de Sevilla, 2012, 1061-1074.

Thiese, Anne-Marie, «Alla base del regionalismo: la definizione della cultura popolare», Nazioni e Regioni, 1, Bari, 2013, 15-20.

Vila, Pep, «Lletres de Mossèn A.M. Alcover i de F.B. Moll a Irene Rocas Romaguera, corresponsal de l'obra del diccionari de Llofriu», Randa, 38, Barcelona, 1997, 147-182. 